\title{
Test-retest reliability of contrast visual acuities in a clinical population
}

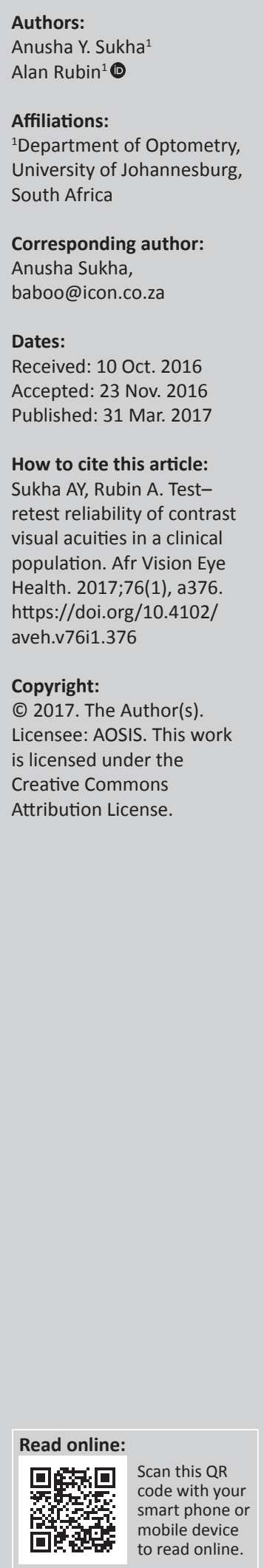

Background: Previously, contrast visual acuities (VA) have been evaluated as a potential screening, diagnostic and predictive tool in cases where standard visual acuity remains intact. Issues around contrast acuity sometimes make it difficult for clinicians to make appropriate clinical decisions and thus such tests have to be standardised and reliable.

Aim: To investigate test-retest reliability of contrast VA in healthy adults in a clinical setting.

Methods: Best compensated contrast VA at $100 \%, 10 \%, 5 \%$ and $2.5 \%$ of 155 patients (mean age $39.7 \pm 12.2$ years) were measured using the computerised Thomson Test Chart 2000 Expert. For all participants and at each contrast level, two measurements per right eye were determined. Test-retest reliability for the four contrast levels were assessed using reliability coefficients and Bland-Altman plots. Participants were also divided into three age groups of young (18-39 years, $n=72$ ), middle-age ( $40-49$ years, $n=45$ ) and elderly (50-67 years, $n=38$ ) and reliability was assessed within and between age and gender groups.

Results: For the whole-sample test and retest, measurements within each contrast level were not statistically different $(p \geq 0.05)$. Thus, test and retest measurements per participant were averaged and whole-sample mean-contrast VA and standard deviations for $100 \%, 10 \%, 5 \%$ and $2.5 \%$ were $-0.146 \pm 0.060,0.050 \pm 0.071,0.135 \pm 0.079$ and $0.405 \pm 0.115 \log$ MAR, respectively. Significant differences were found between all pairs of contrast levels compared $(p \leq 0.0125)$. Mean-contrast VA within each age group were also significantly different across all contrast levels $(p<0.0001)$. Mean-contrast VA at each contrast level between the age groups indicated that mean-contrast VA were not significantly different between the young and middle-age groups $(p>0.05)$ but were statistically different between the young and elderly groups $(p<0.01)$. Only mean-contrast VA $10 \%$ was significantly different between the middle-age and elderly groups $(p<0.001)$. Also, mean-contrast VA for the four contrast levels within gender were significantly different $(p \leq 0.05)$ but not between genders $(p \geq 0.05)$.

Conclusion: This study found good reliability of test and retest measurements of contrast VA in an adult clinical population.

\section{Introduction}

Contrast visual acuities (VA) are an important psychophysical measure of visual or functional dysfunction, and contrast VA are also useful in monitoring the effects of disease-modifying therapies. ${ }^{1,2,3,4,5,6,7,8,9}$ Clinical contrast VA tests, readily available in both printed and computerised charts, are easy to use and generally reliable. . $10,11,12,13,14,15$ Contrast VA measurements at different contrast levels form isolated points on an individual's contrast sensitivity curve, and shifts in these curves become diagnostically important in detecting subtle changes of the visual system, especially in certain pathological conditions where patient's present with $6 / 6$ visual acuity. $2,6,9,10$ Contrast sensitivity testing has been used with ocular diseases (particularly cataract, glaucoma, diabetes mellitus and age-related macular degeneration) and in predicting mobility and functional vision..$_{1,16,17,18,19,20}$ Predominantly, high (100\%) and medium (10\%) contrast levels have been assessed in studies evaluating refractive surgery outcomes, ${ }^{21,22}$ performances of contact lenses ${ }^{4,5,23}$ and activities of daily living. ${ }^{6,17,24}$ For example, Bailey et al. ${ }^{22}$ used $100 \%$ contrast levels and $18 \%$ contrast levels (which they defined as low contrast although $18 \%$ would generally be considered a medium-contrast level) to evaluate the effects of LASIK pre-operatively and 3 and 6 months postoperatively. Low-contrast levels have been evaluated in studies for diagnosing ocular diseases and in monitoring therapeutic interventions. ${ }^{25}$ Recently, with highly active therapy regimes (such as nutritional supplementation, pharmacologic treatments, gene therapy, macular translocation surgery, retinal prosthetic implants, photodynamic therapy or foetal cell transplantation), early detection of ocular complications has become even more critical, as some of the disease complications can be halted or even reversed with these treatments. There are several reports 
showing evidence that during asymptomatic stages of retinal diseases such as diabetic retinopathy or glaucoma (where visual acuity remains good or fairly good), alterations occur in the retinal ganglion cells and in the inner retinal neurons. ${ }^{18,19,25,26,27}$

Contrast sensitivity testing as a potentially valuable clinical measurement has not attained wide acceptance as an additional routine visual screening or examination procedure among optometrists and ophthalmologists, despite the above evidence. A possible reason may be inconsistent results obtained from various studies. For example, studies suggest that contrast sensitivity is affected by many ophthalmic diseases, but not all affected patients show similar changes in contrast sensitivity. 18,19,25,27 Secondly, patients with different ophthalmic diseases may show apparently similar changes in contrast sensitivity. ${ }^{28,29,30}$ However, these discrepancies may also be due to possible limitations or differences in the study methodology (e.g. the use of monocular data in some studies but binocular data in others) and the nature of the contrast sensitivity test itself (charts with different targets or test distances or luminance). Thirdly, the number of spatial frequencies or contrast levels evaluated in studies are not consistent as some authors evaluated only two (mostly high and low) levels, $1,24,26,31,32,33,34$ some three ${ }^{6,35}$ and some four or more. ${ }^{1,15,33,36}$ Other authors have suggested that in most clinical or perhaps even research situations, only one or perhaps two levels of contrast actually need to be measured and methods such as factor analysis have been used to provide support for this assertion. ${ }^{24,26,29,33,34}$

All types of contrast sensitivity letter charts have some advantages for clinicians and patients; they are relatively inexpensive, easy and quick to administer and are generally considered diagnostically sensitive despite some of the limitations already mentioned. ${ }^{1,37,38}$ Not unexpectedly, patients without ocular disease do show some variability in threshold testing but patients with disease may exhibit even greater variability. ${ }^{6,16}$ To aid in efficient clinical management, criteria must be established for objective assessment and comparison of results. Therefore, this study firstly and primarily investigates test-retest reliability of contrast VA measurements (at 100\%, 10\%, 5\% and 2.5\% contrast levels) both within and between contrast levels, and secondly, investigates whether contrast VA measurements, as obtained with a commercially available computer-based test, are perhaps influenced by age or gender. ${ }^{39}$

\section{Methods}

\section{Study population and setting}

One hundred and fifty five $(N=155)$ participants were recruited from a private optometry practice located in Johannesburg, South Africa. Only participants over the age of 18 years were included in the study. Participation was voluntary, and informed and signed consents were obtained. Ethical clearance for the study was obtained (AEC45/01-2011) from the Ethics Committee of the Faculty of Health Sciences of the University of Johannesburg. Only participants without systemic or ocular diseases and without medications, or of ocular surgery or significant vision loss were included. Measurements of all parameters occurred in one clinical environment with the same ambient (mesopic) lighting conditions. Tests were performed in a specific diagnostic sequence and all participants had distance VA of $6 / 6$ or better in each eye with their best compensated subjective refractions.

\section{Procedures}

A basic biographical, general health and ocular history questionnaire was first administered to all participants. The questionnaire concerned demographical aspects (date of birth, age, race and gender), general health and basic systemic conditions (hypertension, respiratory problems, renal conditions, central nervous system conditions or other diseases) and vision-related questions (e.g. if they had any history of ocular surgery and/or diseases, use of medication and headaches). These questions were included to eliminate possible factors that could cause changes in vision involving the eye and retina.

Subjective clinical refractions were performed on all participants to determine best compensated VA for distance (6 m). Stereo-acuity, cover test and Ishihara colour vision tests were administered as additional diagnostic tests to determine if any binocular or colour anomalies were present. Direct ophthalmoscopy and biomicroscopy were used to detect any abnormalities of the ocular media and/or fundi. ${ }^{40}$

\section{Contrast visual acuity assessment}

Contrast VA with their subjective refractions (best compensated VA) were measured with the Thomson Test Chart 2000 Expert software. ${ }^{39}$ This software can generate a wide range of test charts and stimuli, but for this study Bailey-Lovie $\log$ MAR charts with $4 \times 5$ British Sloan letters consisting of, Z, V, P, H, E, F, R, D, U and N were used. Each chart consisted of five of the above letters and a uniform logarithmic progression in size of letters on each line with randomisation of letters as displayed. The contrast of these letters was then reduced from $100 \%$ to $10 \%, 5 \%$ and $2.5 \%$ and measurements were taken accordingly. For statistical analysis, responses were recorded using the 'letter score' method $^{40,41}$ and then converted ${ }^{41}$ to $\log$ MAR scores. The test charts were automatically displayed on a Samsung SyncMaster Series 5, 24 inch widescreen LED monitor, as per the recommendations of the test manufacturer (Thomson), which complies with the European and British standards for adequate display of test charts. ${ }^{39}$

In order to minimise variation of measurements created by the test itself, both the letter size and monitor luminance of the charts were calibrated as follows: (1) To ensure that the letters subtended the correct angle at the eye, we manually confirmed the automatic computerised calibration by calculating and measuring the letter height subtending an angle of 5 min arc at a viewing distance of $4.12 \mathrm{~m}^{40}$ The height of this letter, an 
' $E$ ' in the $6 / 6$ line, was approximately $6 \mathrm{~mm}$ as measured with a millimetre rule. (2) Luminance of the screen target (letter) and background at each of the four different contrast levels were measured using a photospectrometer (the Photo Spectrascan) and averages of three measurements per level were applied to the Weber formula. ${ }^{42}$ For example, at $100 \%$ contrast maximum luminance, Lmax (white ground luminance) $=246.3 \mathrm{cdm}^{-2}$, whereas the minimum luminance, Lmin (black target $/$ stimulus luminance) $=0.8419 \mathrm{cdm}^{-2}$. These measurements with the Weber formula indicated that the contrast was $99.66 \%$ for the $100 \%$ contrast levels, and at $2.5 \%$ the contrast equated to $2.395 \%$.

Two measurements (test and retest) per chart (100\%, 10\%, 5\% and $2.5 \%$ ) for the compensated right eyes of each of the 155 participants were obtained. Before analysis, all data were carefully examined for possible errors, for example, by checking each of the variables for measurements that were out of the expected ranges, and where necessary corrections were made.

\section{Statistical analysis}

The Statistical Consulting Department of the University of Johannesburg assisted with planning and advice regarding data analyses of contrast VA using the Statistical Package for Social Sciences (SPSS, version 21). In some instances, the researchers also used Statistica- or Matlab-based software, for example, for Bland-Altman plots and their analysis. Preliminary analyses were then performed to ensure no violation of the assumptions of normality, linearity and homoscedasticity. ${ }^{43,44}$ Test and retest reliabilities at the various contrast levels were assessed using correlation coefficients, intra-class correlation coefficients (ICCs), coefficients of repeatability (CRs) and limits of agreement (LoA). BlandAltman plots and $\mathrm{LoA}^{45}$ (mean difference \pm 1.96 standard deviation [s.d.]) are commonly used in various optometry and ophthalmology studies to assess agreement - see the dashed lines in Figure 1 for an example of such LoA. Importantly, McAlinden et al. $^{46}$ suggested that confidence intervals (CIs) on such LoA should also be calculated and reported. Thus, here, LoA and their CIs (grey filled intervals surrounding dashed lines in Figure 1 for the upper and lower LoA) were included, together with the respective mean difference or bias (solid black lines in, for example, Figure 1), respectively, in tables and also on the Bland-Altman plots concerned.

Thereafter, the appropriate statistical tests (non-parametric tests, analysis of variance and post hoc tests) were employed to explore the relationships among variables (e.g. differences between test and retest data for the whole sample or for subgroups according to gender and age).

\section{Results}

This clinical sample of 155 participants had a mean age and s.d. of $39.7 \pm 12.2$ years; $80(51.6 \%)$ were male and 75 $(48.4 \%)$ were female, and $73(47.1 \%)$ were of Indian descent.
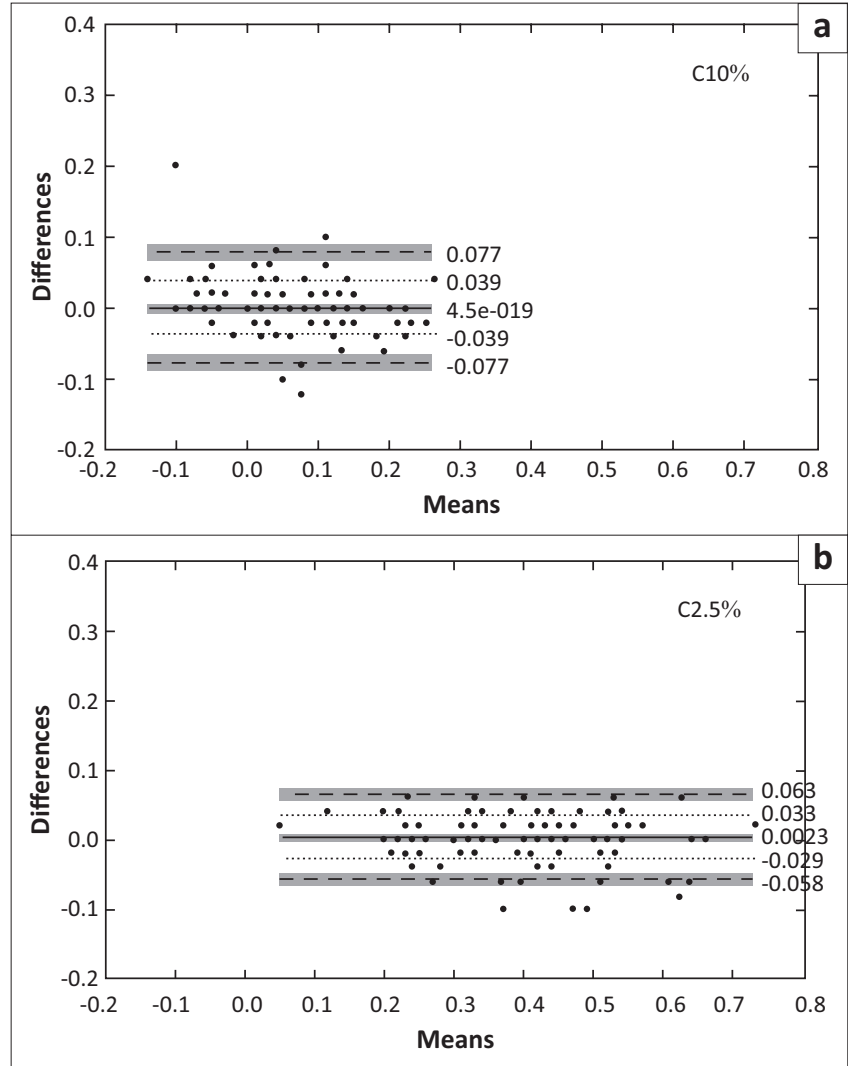

Both Bland-Altman plots have the same scales for easier comparisons. The horizontal solid black lines represent the mean differences and for both contrast levels the mean differences are small and close to zero. The black dotted lines above and below the solid lines on each graph represent the mean difference \pm 1 standard deviation. Two black dashed lines above and below the solid black line on each graph represent the mean difference \pm 1.96 standard deviations, respectively, and represent the upper and lower limits of agreement (LoA). Shaded grey regions indicate $95 \%$ confidence intervals about the mean differences and also the LoA. Irrespective of contrast level, the majority of the points fall within their $95 \%$ LoA. Possible outliers are seen outside the $95 \%$ LoA or sometimes towards the lower or upper limits of the $x$-axis for the means of test and retest data.

FIGURE 1: Bland-Altman plots for the differences (in logMAR) of test and retest measurements against their means (logMAR) for (a) $10 \%$ and (b) $2.5 \%$ contrast levels for the sample of 155 right eyes.

The remaining participants were mostly Caucasian or African. Mean intraocular pressure (and s.d.) were $13 \mathrm{mmHg}$ $\pm 3 \mathrm{mmHg}$ for the right eyes only, and mean clinical refractive status for all (155) right eyes was $-0.70-0.14 \times 7$.

\section{Comparison between test and retest contrast visual acuity measurements}

Table 1 includes descriptive and correlation statistics for the test and retest measurements at the four different contrast levels $(100 \%, 10 \%, 5 \%$ and $2.5 \%)$ for all participants in this study. Hereafter, the samples for a specific contrast level were indicated with the letter $C$ in front of the contrast percentage; thus for $100 \%$ contrast the sample is C100. With Kolmogorov-Smirnov tests, the C100 test and retest samples, the $\mathrm{C} 10$ test and retest samples, the $\mathrm{C} 5$ test and retest samples and also the C2.5 retest samples all had probability values of $p<0.0001$, suggesting departure from data normality $(p<0.05)$. The C2.5 test was the only data displaying normality $(p=0.20)$. As seven of the eight samples here were not normally distributed, mainly non-parametric analyses were employed for further analysis. The mean-contrast VA decreased as contrast levels decreased (from $100 \%$ to $2.5 \%$ ), indicating that participants found it more difficult to correctly 
identify letters at the lower contrast levels (Table 1). Test and retest means for participants varied minimally: by 0.001 $\log$ MAR for contrast $100 \%$ (C100), zero logMAR or 0.08 letters for contrast $10 \%$ (C10), 0.005 logMAR for contrast 5\% (C5) and $0.003 \log$ MAR for contrast $2.5 \%$ (C2.5). The s.d. of the means also increased slightly as contrast decreased; however, contrast VA (and their s.d.) seemed to be fairly consistent when comparing test and retest measurements. The test and retest medians were also very consistent (contrast level 5\% varies by $0.02 \log$ MAR or only one letter). Significant and strong, positive correlations between test and retest measurements for all contrast levels were observed in this study. The correlations for the $2.5 \%$ test and retest measurements were higher when compared with the $100 \%$ level and these results probably may be attributed to participants who generally tended to concentrate more when viewing the more difficult lower contrast letters as compared with the $100 \%$ level which was much easier to read. Another reason may be attributed to truncation of the letter chart where $-0.20 \log$ MAR was the smallest line to be read, thus resulting in a ceiling effect in some eyes where perhaps smaller letters (on the high-contrast chart mainly) might otherwise have been resolved.

Correlation coefficients only measure the strength of a relation between two variables and not the agreement between them. According to Bland and Altman ${ }^{44}$ and McAlinden et al. ${ }^{46}$ repeatability of measurements is

TABLE 1: Means, standard deviations, medians and interquartile ranges for test and retest measurements for contrast visual acuities of the right eyes of 155 participants at contrast levels 100\% (C100), 10\% (C10), 5\% (C5) and 2.5\% (C2.5).

\begin{tabular}{lcccc}
\hline Contrast Level & Mean & s.d. & Median & IQR \\
\hline C100 Test & -0.146 & 0.062 & -0.180 & 0.10 \\
Retest & -0.147 & 0.061 & -0.180 & 0.06 \\
C10 Test & 0.050 & 0.077 & 0.040 & 0.10 \\
Retest & 0.050 & 0.069 & 0.040 & 0.10 \\
C5 Test & 0.138 & 0.082 & 0.140 & 0.08 \\
Retest & 0.133 & 0.079 & 0.120 & 0.06 \\
C2.5 Test & 0.404 & 0.117 & 0.420 & 0.18 \\
Retest & 0.407 & 0.116 & 0.420 & 0.14 \\
\hline
\end{tabular}

Results indicate consistent test and retest measurements and significant positive linear correlations (for Pearson's $p<0.01$ and Spearman's rank-order correlations, $p<0.0005$ ). For $\mathrm{C} 100$, test and retest samples were strongly correlated $(r=0.90, p=0.72)$, and similarly for C10 $(r=0.86, p=0.81), \mathrm{C} 5(r=0.94, p=0.87)$ and C2.5 $(r=0.97, p=0.96)$. Units are logMAR C10 $(r=0.86, p=0.81)$
throughout the table. effectively assessed using the LoA technique and therefore important statistics for the LoA between test and retest measurements for the 155 right eyes and also some measures of repeatability for the four contrast levels are included in Table 2.

The mean-contrast VA (in Table 2) for the whole sample is defined as the overall average of the test and retest measurements, which were themselves averaged across the 155 eyes. Differences were determined by subtracting the test score of each eye from its retest score. In Table 2, the mean-contrast VA and s.d. for contrast level C100 is $-0.147 \pm$ 0.060 ; therefore $-0.147-0.060=-0.207$ or $6 / 3.7$ and $-0.147+$ $0.060=-0.087 \log$ MAR or $\approx 6 / 4.8^{-0.5}$. This indicates the meancontrast VA (-0.147) was approximately $6 / 3.8^{-2.5}$ and about $68 \%$ of the measurements were between $6 / 3.8^{-2.5}$ and $6 / 4.8^{-0.5}$, and $95 \%$ would be between the mean \pm 1.96 s.d. Using letter count, the s.d. approximates to about three letters for $\mathrm{C} 100$, about 3.5 letters for $\mathrm{C} 10$, about four letters for $\mathrm{C} 5$ and about five letters for $\mathrm{C} 2.5$, indicating that variation in contrast VA measurements increases slightly as contrast levels decrease. The mean differences between 155 test and retest measurements were small and almost zero, irrespective of contrast level. The s.d. of the mean differences in letters ranged from 1.5 to 2 letters or $0.03-0.04 \log$ MAR, indicating minimal variation between the test and retest measurements. Individual right eyes might, however, have shown larger differences between test and retest measurements.

With all contrast levels, the standard errors (see Table 2) for the respective mean differences are small. Generally, LoA are also small and the $95 \%$ CIs for the mean differences and for the upper and lower LoA are relatively narrow, indicating that the mean differences and the interval estimates are very precise.

The four CRs are almost zero, and the ICCs in Table 2 for all contrast levels are approximately 1, indicating high levels of consistency or reliability between test and retest measurements for each of the four contrast levels. These results suggest little in the way of learning or fatigue effects with these participants. Of these, $\mathrm{C} 10$ had the highest $\mathrm{CR}$ value of $\approx 0.08$, indicating that test and retest measurements

TABLE 2: Descriptive statistics and limits of agreement between the test and retest measurements for 155 right eyes for four contrast levels, namely $100 \%, 10 \%, 5 \%$ and $2.5 \%$. Standard errors and confidence intervals for mean differences $\left(\bar{\chi}_{d}\right)$ and LoA are also included. Measures of repeatability such as coefficients of repeatability and intra-class correlation coefficients are also provided.

\begin{tabular}{|c|c|c|c|c|}
\hline$N=155$ & $100 \%$ & $10 \%$ & $5 \%$ & $2.5 \%$ \\
\hline Mean-contrast VA (s.d.) & $-0.147(0.060)$ & $0.050(0.071)$ & $0.135(0.079)$ & $0.405(0.116)$ \\
\hline Mean differences $\left(\bar{\chi}_{d}\right)$ (s.d.) & $-0.000(0.027)$ & $0.000(0.040)$ & $-0.005(0.0286)$ & $0.002(0.031)$ \\
\hline Standard error for $\bar{\chi}_{d}$ & 0.002 & 0.003 & 0.002 & 0.003 \\
\hline $95 \% \mathrm{Cl}$ for $\bar{\chi}_{d}$ & $-0.005 ; 0.004$ & $-0.006 ; 0.006$ & $-0.009 ;-0.000$ & $-0.003 ; 0.007$ \\
\hline LoA: $\bar{\chi}_{d} \pm 1 s d$ & $-0.027 ; 0.027$ & $-0.039 ; 0.039$ & $-0.033 ; 0.024$ & $-0.029 ; 0.033$ \\
\hline $\bar{\chi}_{d} \pm 1.96 s d$ & $-0.055 ; 0.053$ & $-0.077 ; 0.077$ & $-0.062 ; 0.052$ & $-0.058 ; 0.063$ \\
\hline Standard error of LoA & 0.004 & 0.006 & 0.004 & 0.004 \\
\hline $\mathrm{Cl}$ of upper $\mathrm{LoA}$ & $0.045 ; 0.060$ & $0.067 ; 0.088$ & $0.043 ; 0.059$ & $0.054 ; 0.071$ \\
\hline $\mathrm{Cl}$ of lower LoA & $-0.062 ;-0.047$ & $-0.088 ;-0.067$ & $-0.069 ;-0.053$ & $-0.067 ;-0.050$ \\
\hline $\mathrm{CR}$ & 0.053 & 0.077 & 0.057 & 0.061 \\
\hline ICC & 0.904 & 0.857 & 0.935 & 0.965 \\
\hline
\end{tabular}


were slightly less similar, but the presence of outliers were important in this sample and may have influenced the result (see Figure 1a where four measurements were outside the 95\% LoA).

For simplicity, Figure 1 includes only two (10\% and $2.5 \%)$ of the four contrast levels, but all Bland-Altman plots revealed that the majority of the points were located relatively near to the solid black line representing the applicable mean difference for the contrast level concerned. Most points for each contrast level were located within their $95 \%$ LoA, and so largely irrespective of contrast level the variability of the differences in test and retest measurements was small: about 1.5 letters (approximately $0.03 \log \mathrm{MAR}$ ) for C100, C5 and C2.5; and two letters (or $0.04 \log \mathrm{MAR}$ ) for C10\%; thus, only two plots are provided here (see Figure 1). The Bland-Altman plots indicated that the distributions of the differences between the test and retest contrast VA scores for all four contrast levels reflected minimal variability and therefore generally the test and retest scores can be considered to be in good agreement.

Wilcoxon signed-rank tests for the four paired comparisons (i.e. between test and retest measurements for C100, C10, C5 and C2.5) revealed that for all contrast levels, test and retest contrast VA were not statistically different $(p \geq 0.05)$, suggesting that there were minimal learning and fatigue effects (or that these two effects cancelled each other out). Consequently, for each eye and for all four contrast levels, corresponding test and retest contrast VA measurements were averaged resulting in one sample of Mean-contrast VA per contrast level. These four new samples of Mean-C100, Mean-C10, Mean-C5 and Mean-C2.5 ( $N=155$ for each sample) were used for further analysis instead of

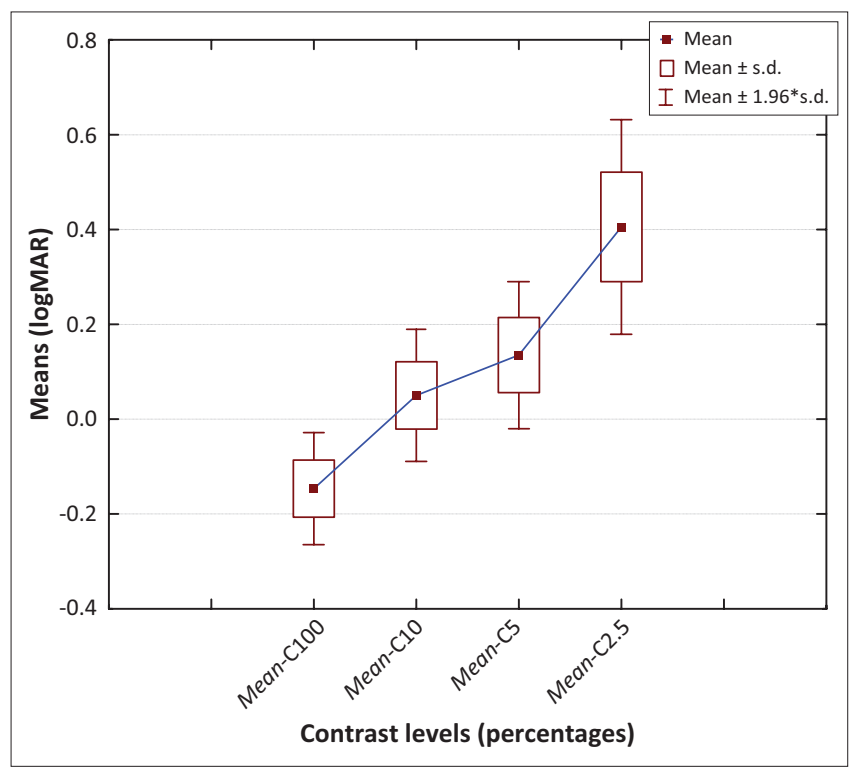

The sample means are $-0.147 \log$ MAR (for Mean-C100 or $100 \%$ contrast) and 0.050 logMAR (for Mean-C10) and 0.135 logMAR (for Mean-C5) and 0.405 logMAR (for Mean-C2.5). A statistically significant difference in the means (small squares in the figure) for Meanstatistically significant difference in the means (small squares in the figure) for Mean-
contrast VA for the four contrast levels was found after a Bonferroni correction (to $p<$ contrast VA for the four contrast levels was found after a Bonferroni correction (to
0.0125 ) was applied. The whiskers represent the mean \pm 1.96 s.d. for specific samples.

FIGURE 2: Box and whisker plots are used to compare samples of mean-contrast VA (solid small squares) at four different contrast levels $(100 \%, 10 \%, 5 \%$ and $2.5 \%$ ) for 155 right eyes. the eight test and retest samples and this simplifies such an analysis.

Using the Friedman's chi-squared test, a statistically significant difference in Mean-contrast VA for $100 \%, 10 \%, 5 \%$ and $2.5 \%$ contrast levels was found $\left(\chi_{3,155}^{2}=460.98, p<0.0001\right.$, Mean-C100 -0.147 $\pm 0.060 \operatorname{logMAR}$; Mean-C10 $0.050 \pm 0.071$ $\log$ MAR; Mean-C5 $0.135 \pm 0.079 \log$ MAR and Mean-C2.5 $0.405 \pm 0.116 \log \mathrm{MAR})$. Thereafter, post hoc comparisons were conducted to determine which Mean-contrast VA pairs were significantly different from one other based on the mean ranked differences of each. The Wilcoxon signed-rank test was performed and a Bonferroni correction was applied (the alpha level, usually 0.05 , was divided by the number of variables [four here because of the four different contrast levels]). Thus, $p=0.05 / 4=0.0125$ and all pairs of samples of Mean-contrast VA compared (Figure 2) were statistically different $(p<0.0125)$.

\section{Comparison of contrast visual acuities means within and between genders}

The study sample consisted of 80 males (mean age and s.d. of $39.67 \pm 12.48$ years) and 75 females (mean age and s.d. of $39.73 \pm 12$ years). Within gender, Friedman tests indicated that mean-contrast levels within the males and also within the females were significantly different across the four contrast levels: for males, $\chi_{3,80}^{2}=237.13, p<0.0001$; and for females, $\chi_{3,75}^{2}=223.86, p<0.0001$. With post hoc tests and the Wilcoxon signed-rank test with a Bonferroni correction of $p=0.013$, it was found for both the males and females that within each gender, there were statistically significant differences between the various contrast levels.

Mann-Whitney $U$ tests revealed that means for the averaged test and retest contrast measurements for all levels were not significantly different (i.e. $p>0.05$ ) between males and females (Mean-C100 $z=-1.416, p=0.16$; Mean-C10 $z=-0.505$, $p=0.61$; Mean-C5 $z=-0.506, p=0.61$; and Mean-C2.5 $z=-0.973$, $p=0.33)$.

\section{Comparison of contrast visual acuities means between different age groups}

The 155 participants were divided into three age groups consisting of young pre-presbyopic adults $(n=72)$ of age 18-39 years, middle-age adults $(n=45)$ of age $40-49$ years and elderly $(n=38)$ of age $50-67$ years. The Friedman test found significant differences $(p<0.05)$ for mean-contrast VA level within each age group: in the young age group $\chi_{3,72}^{2}=$ $214.535, p<0.0001$; in the middle-age group $\chi_{3,45}^{2}=133.897$, $p<0.0001$; and in the elderly group $\chi_{3,38}^{2}=112.567, p<0.0001$. These analyses indicated that within each age group, contrast VA at the four contrast levels $100 \%, 10 \%, 5 \%$ and $2.5 \%$ were significantly different from each other.

Box and whisker plots in Figure 3 are used to graphically compare Mean-contrast VA of the three age groups at the four contrast levels as investigated. The Kruskal-Wallis analysis of variance revealed that Mean-contrast VA varied significantly 
$(p<0.05)$ across all age groups for all contrast levels: Mean-C100 $\chi_{2,155}^{2}=10.390, p=0.01 ;$ Mean-C10 $\chi_{2,155}^{2}=15.535$, $p<0.0001 ;$ Mean-C5 $\chi_{2,155}^{2}=11.998, p<0.01$ and Mean-C2.5 $\chi_{2,155}^{2}=11.161, p<0.01$.

Post hoc tests were conducted to determine for which age groups the mean (contrast VA) differences were present. The Mann-Whitney $U$ test was used to test for these differences and Bonferroni adjustments (to set more stringent alpha values because of the number of tests and samples) were made. $^{47}$

The Mann-Whitney U analyses tested the following three pairs:

- Pair 1: Mean-C100, C10, C5 and C2.5 in the young versus the middle-age group.

Results indicated that Mean-C100 $(z=-2.01, p=0.04)$, Mean-C10 $(z=-1.37, p=0.17)$, Mean-C5 $(z=-1.82, p=0.07)$ and Mean-C2.5 $(z=-2.08, p=0.04)$ were not significant between the young and middle-age groups $(p>0.03,0.05$, 0.03 and 0.02 , respectively).

- Pair 2: Mean-C100, C10, C5 and C2.5 in the young versus the elderly group.

Results indicated that Mean-C100 $(z=-3.00, p<0.01)$, Mean-C10 ( $z=-3.90, p<0.0001)$, Mean-C5 $(z=-3.38$, $p<0.01)$ and Mean-C2.5 $(z=-3.28, p<0.01)$ were significantly different between the young and elderly groups ( $p<0.05,0.002,0.03$ and 0.01 , respectively).

- Pair 3: Mean-C100, C10, C5 and C2.5 in the middle-age versus the elderly group.

Results indicated Mean-C100 $(z=-1.48, p=0.14)$, Mean-C5 $(z=-1.58, p=0.11)$ and Mean-C2.5 $(z=-1.15, p=0.25)$ were not significantly different between the middle-age and elderly groups ( $p>0.03,0.03$ and 0.05 , respectively).

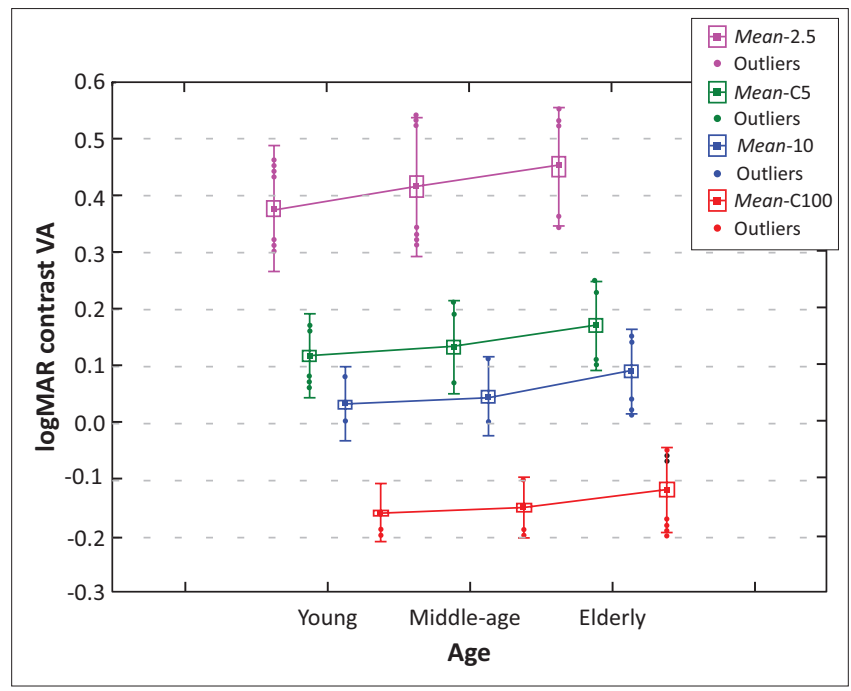

The $y$-axis is contrast VA in logMAR. Statistically significant differences for all levels of contrast VA were found between the young age and elderly groups (for all contrast levels the contrast VA were found between the young age and elderly groups (for all contrast levels the blue line is below the green line) and for Mean-C10 contrast VA between the middle-age an elderly groups but not for Mean-C100, Mean-C5 or Mean-C2.5. The small solid square whiskers represent the mean \pm 1 standard deviation.

FIGURE 3: Box and whisker plots are used for comparison of the Mean-contrast visual acuities between young (18-39 years, $n=72$ ), middle-age ( $40-49$ years, $n=45$ ) and elderly (50-67 years, $n=38$ ) age groups.
Mean-C10 contrast VA in the middle-age group was significantly different $(p<0.02)$ to the elderly group $(z=-2.49, p=0.01)$.

In conclusion, the results indicated that means (of test and retest) contrast VA at each of the four levels were significantly different between the young and elderly eyes, but not between the young and middle-age eyes. For the middle-age and elderly eyes, only Mean-contrast VA at the $10 \%$ level was different at a $95 \%$ level of confidence. ${ }^{47}$

\section{Discussion}

The results of this study for the whole sample (155 right eyes), firstly, suggest that contrast VA at each of the levels of $100 \%, 10 \%, 5 \%$ and $2.5 \%$ demonstrated no significant statistical differences between test and retest measurements. Thus, such measurements with the computerised method were consistent and repeatable. Secondly, once test and retest contrast VA for each contrast level for each participant were averaged, statistically significant differences for the whole sample across the four contrast levels were found. That is, as contrast level reduces from $100 \%$ to $2.5 \%$, mean-contrast VA for the group decreases. Thirdly, there were no real differences found between the two genders for either contrast VA means or their variations, and thus, test and retest measurements were consistent both within and across gender. Analyses of mean-contrast VA between age groups indicated that variability in responses was consistent at all four contrast levels. Means were also consistent between the younger and middle-age groups, and between the elderly and middle-age groups. However, in high-, medium- and low-contrast VA, means were found to be statistically different between the young (18-39 years) and elderly (50-67 years) age groups, despite the differences in sample sizes (72 vs. 38 ).

Direct comparisons of our results to other publications are difficult to make because of the different clinical and statistical methodologies and analyses used between studies. However, Ehrmann et al. ${ }^{12}$ also found test and retest measurements for the Thomson Test Chart 2000 PRO (an earlier software version to the one that we used) to be very repeatable in terms of $100 \%$ and $10 \%$ contrast VA. The Thomson Test Chart was used to cross-validate $100 \%$ and $10 \%$ contrast VA data using traditional Bailey-Lovie paper charts and the Thomson Test Chart 2000 PRO. In their study, habitual contrast VA was measured both monocularly and binocularly at $6 \mathrm{~m}$ in 40 healthy adult subjects (mean age of 36 years compared with 155 subjects with a mean age of 39.7 years in this study). Using the VA group data for the computerised measurements for right eyes only (monocular) from their study, the means and s.d. for $100 \%$ and $10 \%$ contrast VA were $-0.02 \pm 0.10$ $\log$ MAR and $0.22 \pm 0.13 \log$ MAR, respectively. In our study, contrast VA for both these contrast levels were found to be better and the means and s.d. for $100 \%$ and $10 \%$ contrast VA were $-0.147 \pm 0.06 \log$ MAR and $0.050 \pm 0.07 \log$ MAR, respectively. Thus, the difference of means (between the two studies) for $100 \%$ and $10 \%$ equates to 0.123 and $0.17 \log$ MAR, respectively, corresponding to a six letter difference for $100 \%$ 
and an eight and half letter difference for 10\% contrast VA. The difference in s.d. for both contrast levels is only about two letters (0.04 logMAR). Thus, the monocular results from Ehrmann et al. ${ }^{12}$ for the means are somewhat different to results obtained from our study. The better contrast VA obtained from this study is probably attributed to measuring contrast VA with optimal refractive compensation (and VA better than $0 \log$ MAR) when compared with Ehrmann et al. ${ }^{12}$ where contrast VA was obtained with habitual vision (a minimum VA $6 / 12$ for $100 \%$ contrast and $6 / 19$ for $10 \%$ contrast). When comparing the binocular computerised VA group data from Ehrmann et al. ${ }^{12}$ with means for contrast VA results from our study, similar or more consistent results were found (the binocular mean and s.d. for $100 \%$ and $10 \%$ contrast VA were $-0.08 \pm 0.08 \log$ MAR and $0.09 \pm 0.09$ $\log \mathrm{MAR}$, respectively). Here the difference in means equates to $0.06 \log$ MAR (or three letters) and $0.04 \log$ MAR (or two letters) for 100 and 10\%, respectively. The differences in s.d. for each contrast level were only one letter $(0.02 \log M A R)$. A maximum of a three and a half letter difference for means and a one letter difference for s.d. may be considered small; thus, in this comparison results are found to be consistent. Therefore, the Bland-Altman plot for the binocular 100\% contrast VA for the monitor chart included by Ehrmann et al. ${ }^{12}$ can be compared with the $95 \%$ LoA results for the $100 \%$ contrast level from our study. For their sample, the mean difference was almost zero (0.0004 $\log$ MAR) and the LoA were 0.073 and $-0.065 \log$ MAR. These results are similar to those in our study (a mean difference of $-0.0003 \log$ MAR and for the $95 \%$ LoA upper and lower limits of 0.053 and -0.055 $\log$ MAR). The slightly smaller or narrower (and thus more reliable) LoA found in our study may also be attributed to the reasons previously mentioned; that is, in our study a larger sample size was used and contrast VA were obtained with best compensated refractions rather than subjects using their habitual compensations or none at all. ${ }^{12}$ In conclusion, results obtained from our study are found to be fairly consistent with the binocular results from Ehrmann et al. ${ }^{12}$ in terms of contrast VA means and s.d. for the $100 \%$ and $10 \%$ contrast levels and in terms of repeatability for the $100 \%$ contrast level.

Further comparisons of $100 \%$ and $2.5 \%$ contrast VA from a cross-sectional observational study by Pineles et al. ${ }^{20}$ with disease-free controls $(n=324)$ can be made to our study. Their $100 \%$ mean-contrast VA (measured with the ETDRS chart at $3.2 \mathrm{~m}$ ) compares our results with only a two letter difference. Their 2.5\% mean-contrast VA (measured with a Sloan chart at $2 \mathrm{~m}$ ) compares with a three letter difference in our study. In their study, the mean age was $40 \pm 11$ years, whereas in ours, the mean age was $39.7 \pm 12.2$ years; in our study, all levels of contrast VA were tested using the Bailey-Lovie Sloan chart at $6 \mathrm{~m}$. So, different test distances and charts are possible factors that may have led to the slight differences in contrast VA of the two studies concerned.

Results from our study are consistent with studies which reported no association of gender with contrast VA. ${ }^{15,48}$
The Smith-Kettlewell Eye Research Institute also evaluated gender differences with regard to night driving selfrestrictions and vision function in an elderly population of 376 males and females. The vision tests evaluated subjects' binocular habitual corrections with the Bailey-Lovie high (90\%) and medium or low (18\%) contrast VA charts, the PelliRobson contrast sensitivity chart, the low-contrast, lowluminance acuity SKILL cards, the Frisby stereo test and the Berkeley glare test. ${ }^{33}$ For the contrast sensitivity and highand low-contrast VA tests, small but statistically significant differences (gender in ANOVA) were found between men and women drivers on low- (or medium-) contrast VA (18\%) and contrast sensitivity only with women having slightly better vision than men. Possible explanations for the differences found in contrast VA for low contrast between genders and between the studies are unclear but perhaps the use of different contrast charts or other physical (e.g. foveal density) or psychological factors of the individuals concerned may be possible factors that contribute to this disparity.

The distribution of mean $100 \%$ contrast VA within a large, clinically healthy population ${ }^{49}$ generally correlates with our results. Comparisons of $100 \%$ contrast VA could be made with results of Elliot et al. ${ }^{49}$ in 223 subjects. Their meancontrast VA compared well with our mean $100 \%$ contrast VA in the respective age groups. The comparisons demonstrated that in their young group (18-39 years) mean VA of -0.145 $(n=77)$ was similar to our $-0.159 \log$ MAR $(n=72)$, essentially only a single (1) letter mean difference. In their middle-age group (40-49 years), they found mean VA of $-0.125(n=40)$ compared with our $-0.149 \log$ MAR $(n=45)$, also signifying only a single letter difference on average between the two studies. However, in their elderly group (50-67 years), they found mean VA of $-0.08(n=63)$ compared with our -0.1187 $\log \operatorname{MAR}(n=38)$ or a two letter (mean) difference.

The means and s.d. of the younger group from our study for $100 \%(-0.16 \pm 0.05 \log \mathrm{MAR})$ and $10 \%(0.03 \pm 0.06 \log \mathrm{MAR})$ contrast VA can also be compared with results from Hazel and Elliott ${ }^{50}$ (mean age of 28 years, with VA $>6 / 6$ ). Test and retest habitual high-contrast VA were evaluated ${ }^{50}$ monocularly (using letter-by-letter scoring) using four charts, namely the Bailey-Lovie, ETDRS, Regan and Waterloo, and low-contrast VA Bailey-Lovie $10 \%$ and Regan $11 \%$ contrast charts in 40 healthy subjects (mean age of 28.4 years, with VA $>6 / 6$ ). As in the current study, their study also indicated that there were no significant learning effects from test to retest scores $(p>0.10)$, but a significant difference between the visual acuity scores from the four high-contrast logMAR charts was found. In addition, post hoc tests indicated better letter contrast VA from the Regan chart in comparison with the others which was attributed to the letter font type used. The repeatability of the high-contrast charts were similar with ICCs between test and retest data: $0.80,0.79,0.84$ and 0.83 for high Bailey-Lovie, high EDTRS, high Regan and high Waterloo charts, respectively. For low-contrast charts, ICCs were 0.77 for the Bailey-Lovie $10 \%$ chart, and 0.84 for the Regan 11\% chart. The ICCs for test and retest data with 
the Thomson chart in our study showed slightly better repeatability for $100 \%$ and $10 \%$ contrast (ICCs of 0.90 and 0.86 , respectively). These small differences in results may be attributed to chart fonts and chart designs.

\section{Conclusion}

The results from our study established good test-retest reliability of Thomson computer-based contrast VA charts and provide the statistical limits of contrast VA in a clinically healthy population, without serious visual impairment. These results also re-emphasise the need to measure VA and subsequently contrast VA, to threshold for different contrast levels and for different age groups. These can in turn be used for comparison with eyes, where a reduced level of contrast VA may have pathological or diagnostic implications. The results and methods in this study provide support for the use of a computerised and potentially useful clinical and research tool and may be used to diagnose, investigate and monitor ocular or systemic diseases with ocular manifestations, or with pre- and post-operative refractive surgery. They can also be used in other ocular investigations such as in vision therapy for amblyopia where contrast VA measurements have been found to be a more sensitive detector of visual function.

\section{Acknowledgements Competing interests}

The authors declare that they have no financial or personal relationships that may have inappropriately influenced them in writing this article.

\section{Authors' contributions}

Results are from a doctorate dissertation by A.Y.S. and A.R. was the supervisor.

\section{References}

1. Balcer LJ, Galetta SL, Polman $\mathrm{CH}$, et al. Low-contrast acuity measures visua improvement in phase 3 trial of natalizumab in relapsing MS. J Neurol Sci [serial on the Internet]. 2012;318:119-124 [cited 2013 Mar]. Available from: http:// on the Internet]. 2012;318:119-124 [cited 2013 Mar]. A
www.jns-journal.com/article/S0022-510X(12)00150-5/pdf

2. Balcer LJ, Frohman EM. Evaluating loss of visual function in multiple sclerosis as measured by low-contrast letter acuity. Neuro [serial on the Internet]. 2010;74:S16-S23 [cited 2013 Mar]. Available from: http://www.neurology.org/ content/74/17_Supplement_3/S16.full.pdf+html

3. Oomachi K, Ogata K, Sugawara T, Hagiwara A, Hata A, Yamamoto S. Evaluation of contrast visual acuity in patients with retinitis pigmentosa. Clin Ophthalmol [serial on the Internet]. 2011;5:1459-1463 [cited 2013 Mar]. Available from: https:// www.dovepress.com/evaluation-of-contrast-visual-acuity-in-patients-withWWW.dovepress.com/evaluation-of-contrast-
retinitis-pigmen-peer-reviewed-article-OPTH

4. Rae SM, Allen PM, Radhakrishnan $\mathrm{H}$, et al. Increasing negative spherical aberration with soft contact lenses improves high and low contrast visual acuity in young adults. Ophthalmic Physiol Opt. 2009;29:593-601. https://doi.org/10.1111/j. 1475-1313.2009.00678.x

5. Pettersson AL, Martensson L, Salkic J, Unsbo P, Brautaset R. Spherical aberration in relation to visual performance in contact lens wear. Con Lens Anterior Eye. 2011;34:12-16. https://doi.org/10.1016/j.clae.2010.08.008

6. Sukha AY, Rubin A. High, medium and low contrast visual acuities in diabetic retinal disease. Optom Vis Sci. 2009;86:1086-1095. https://doi.org/10.1097/ OPX.0b013e3181b48635

7. Schneck ME, Haegerstrom-Portnoy G, Lott LA, Brabyn JA, Gildengorin G. Low contrast vision function predicts subsequent acuity loss in an aged population: The SK study. Vision Res. 2004;44:2317-2325. https://doi.org/10.1016/j.visres.2004.04.018

8. Elliott DB, Situ P. Visual acuity versus contrast sensitivity in early cataract. Vision Res. 1998;38:2047-2052. https://doi.org/10.1016/S0042-6989(97)00382-9
9. Balcer LJ, Baier ML, Pelak VS, et al. New low-contrast vision charts: Reliability and test characteristics in patients with multiple sclerosis. Mul Scler. 2000:6:163-171. https://doi.org/10.1177/135245850000600305

10. Colenbrander A, Fletcher DC. The mixed contrast reading card, a new screening test for contrast sensitivity. Inter Congress Series. 2005;1282:492-497. https:// doi.org/10.1016/j.ics.2005.05.212

11. Lovie-Kitchen JE, Brown B. Repeatability and intercorrelations of standard vision tests as a function of age. Optom Vis Sci [serial on the Internet]. 2000;77:412-420 [cited 2013 Mar]. Available from: http://journals.Iww.com/optvissci/toc/2000/08000

12. Ehrmann $K$, Fedtke $C$, Radic A. Assessment of computer generated vision charts. Con Lens Anterior Eye. 2009;32:133-140. https://doi.org/10.1016/j.clae.2008.09.005

13. Lam AKC, Tong P, Tse J, Yu M. Repeatability of near visual acuity measurement at high and low contrast. Clin Exp Optom. 2008;91:447-452. https://doi. org/10.1111/j.1444-0938.2007.00235.x

14. Beck RW, Moke PS, Turpin AH, et al. A computerized method of visual acuity testing: Adaptation of the early treatment of diabetic retinopathy study testing testing: Adaptation of the early treatment of diabetic retinopathy study testing
protocol. Am J Ophthalmol. 2003;135:194-205. https://doi.org/10.1016/s0002 9394(02)01825-1

15. Khanani AM, Brown SM, Xu KT. Normal values for a clinical test of letter recognition contrast thresholds. J Cataract Refract Surg. 2004:30:2377-2382. https://doi. org/10.1016/j.jcrs.2004.05.027

16. Sukha AY, Rubin A. Short-term variation of autorefraction in diabetic macular edema. S Afr Optom. 2007;66:150-162.

17. Fletcher DC, Schuchard RA. Visual function in patients with choroidal neovascularisation resulting from age-related macular degeneration: The importance of looking beyond visual acuity. Optom Vis Sci. 2006;83:178-189. https://doi.org/10.1097/01.opx.0000204510.08026.7f

18. Shah AS, Gundel R. Low-contrast visual acuity measurements in single vision and bifocal soft lens wearers. Inter Contact Lens Clinic. 2000;27:119-123. https://doi. org/10.1016/S0892-8967(02)00067-6

19. Dosso AA, Bonvin YM, Morel Y, Golay A, Assal JP, Leuenberger PM. Risk factors associated with contrast sensitivity loss in diabetic patients. Graefe's Arch Clin Exp Ophthalmol. 1996;234:300-305. https://doi.org/10.1007/BF00220704

20. Pineles SL, Birch EE, Talman LS, et al. One eye or two: A comparison of binocular and monocular low-contrast acuity testing in multiple sclerosis. Am J Ophthalmol. 2011;155:133-140. https://doi.org/10.1016/j.ajo.2011.01.023

21. Hansraj R, Oduntan AO, Rasengane TA. Visual acuity, contrast sensitivity and subjective perception of vision after LASIK refractive surgery. S Afr Optom. 2006;65:12-26.

22. Bailey MD, Olson MD, Bullimore MA, Jones L, Maloney RK. The effect of LASIK on best corrected high and low contrast visual acuity. Optom Vis Sci [serial on the Internet]. 2004;81:362-368 [cited 2013 Mar]. Available from: http://journals.Iww. com/optvissci/toc/2004/05000

23. Vaz TC, Gundel RE. High and low contrast visual acuity measurements in spherical and aspheric soft contact lens wearers. Cont Lens Anterior Eye. 2003;26:147-151. https://doi.org/10.1016/S1367-0484(03)00025-0

24. Stifter E, Sacu S, Thaler A, Weghaupt H. Contrast acuity in cataracts of different morphology and association to self-reported visual function. Invest Ophthalmo Vis Sci. 2006;47:5412-5422. https://doi.org/10.1167/iovs.05-1564

25. Misra S, Saxena S, Kishore P, Bhasker SK, Misra A, Meyer CG. Association of contrast sensitivity with logMAR visual acuity and glysosylated haemoglobin in non-insulin dependent diabetes mellitus. J Ocul Biol Dis Inform. 2010;3:60-63. https://doi.org/10.1007/s12177-010-9056-0

26. Puell MC, Barrio AR, Palomo-Alvarezc, Gomez-Sanz FJ, Clement-Corral A, PerezCarasco MJ. Impaired mesopic visual acuity in eyes with early age-related macular degeneration. Invest Ophthalmol Vis Sci. 2012;53:7310-7314. https://doi. org/10.1167/iovs.11-8649

27. Wood JM, Lovie-Kitchin JE. Evaluation of the efficacy of contrast sensitivity measures for the detection of early primary open-angle glaucoma. Optom Vis $\mathrm{Sci}$ measures for the detection of early primary open-angle glaucoma. Optom Vis Sci
[serial on the Internet]. 1992;69:175-181 [cited 2013 Mar]. Available from: [serial on the Internet]. 1992;69:175-181 [cited
http://journals.Iww.com/optvissci/toc/1992/03000

28. Woods RL, Tregear SJ, Mitchell RA. Screening for ophthalmic disease in older subjects using visual acuity and contrast sensitivity. Ophthalmology. 1998;105:2318-2326. https://doi.org/10.1016/S0161-6420(98)91235-0

29. Elliott DB, Whitaker D. How useful are contrast sensitivity charts in optometric practice? Case reports. Optom Vis Sci [serial on the Internet]. 1992;69:378-385 [cited 2013 Mar]. Available from: http://journals. Iww.com/optvissci/toc/1992/05000

30. Mitchell RA. Contrast sensitivity in elderly subjects with a diagnosed ocular disease. Optom Vis Sci [serial on the Internet]. 1993;70:102-106 [cited 2013 Mar]. Available from: http://journals.Iww.com/optvissci/toc/1993/02000

31. Brabyn J, Schneck ME, Haegerstrom-Portnoy G. Night driving self-restriction Vision function and gender differences. Optom Vis Sci [serial on the Internet] 2005;82:755-764 [cited 2013 Mar]. Available from: http://journals.Iww.com/ optvissci/toc/2005/08000

32. Haegerstrom-Portnoy G, Schneck ME, Lott LA, Brabyn JA. The relation between visual acuity and other spatial vision measures. Optom Vis Sci [serial on the Internet]. 2000;77:653-662 [cited 2013 Mar]. Available from: http://journals.Iww. com/optvissci/toc/2000/12000

33. Reeves BC, Wood JM, Hill AR. Reliability of high- and low-contrast letter charts. Ophthal Physiol Opt. 1993;13:17-26. https://doi.org/10.1111/j.1475-1313.1993. tb00421.x

34. Brown B, Lovie-Kitchin JE. High and low contrast acuity and clinical contrast sensitivity tested in a normal population. Optom Vis Sci [serial on the Internet]. 1989;66:467-473 [cited 2013 Mar]. Available from: http://journals.Iww.com/ optvissci/toc/1989/07000 
35. Saidha S, Syc SB, Durbin MK, et al. Visual dysfunction in multiple sclerosis correlates better with optical coherence tomography derived estimates of macular ganglion cell layer thickness than peripapillary retinal nerve fiber layer thickness. Multi Scler. 2011;17:1449-1463.

36. Miyajima H, Katsumi O, Wang GJ. Contrast visual acuities in cataract patients. Comparison with normal subjects. Acta Ophthalmol. 1992;70:44-52.

37. Bradley DE, Flom RE, Bullimore MA. An evaluation of the MARS letter contrast sensitivity test. Optom Vis Sci [serial on the Internet]. 2005;82:970-975 [cited 2013 Mar]. Available from: http://journals.Iww.com/optvissci/toc/2005/11000

38. Woods RL, Wood JM. The role of contrast sensitivity charts in clinical practice. Clin Exp Optom [serial on the Internet]. 1995;78:43-55 [cited 2013 Mar]. Available from: http://eprints.qut.edu.au/11919/

39. [cited 2014 Jul 01]. Available from: http://www.thomson-software-solutions.com/

40. Benjamin WJ. Borish's clinical refraction. 2nd ed. Philadelphia, PA: ButterworthHeinemann; 2006; p. 210-227.

41. International Council of Ophthalmology. Visual standards: Aspects and ranges of vision loss with emphasis on population surveys [homepage on the Internet] Sydney: ICO; 2002 [cited 2013 Mar]. Available from: http://www.icoph.org/

42. Norton TT, Corliss DA, Bailey JE. The psychophysical measurement of visual function. Woburn: Butterworth Heinemann, 2002

43. Freund JE. Modern elementary statistics. 10th ed. Upper Saddle River, NJ: Prentice Hall; 2000.
44. Pallant J. SPSS survival manual. 2nd ed. Chicago, IL: Open University Press; 2005.

45. Bland JM, Altman DG. Statistical methods for assessing agreement between two methods of clinical measurement. Lancet. 1986: i307-310. https://doi. org/10.1016/S0140-6736(86)90837-8

46. McAlinden C, Khadka J, Pesudovs K. Statistical methods conducting agreement (comparison of clinical tests) and precision (repeatability or reproducibility) studies in optometry and ophthalmology. Ophthalmic Physiol Opt. 2011;31:330338. https://doi.org/10.1111/j.1475-1313.2011.00851.x

47. Sukha AY. Inter-and intra-subject variation of contrast visual acuities [homepage on the Internet. PhD (Optometry): University of Johannesburg; 2015 [cited 2013 Mar]. Available from: http://hdl.handle.net/10210/14030

48. Rubin GS, West SK, Munoz B, et al. A comprehensive assessment of visual impairment in a population of older Americans. Invest Ophthalmol Vis Sci [serial on the Internet]. 1997;38:557-568 [cited 2013 Mar]. Available from: http://iovs. arvojournals.org/article.aspx?articleid $=2161347$

49. Elliot DB, Yang KCH, Whitaker D. Visual acuity changes throughout adulthood in normal, healthy eyes: Seeing beyond 6/6 [serial on the Internet]. Optom Vis Sci. 1995;72:186-191 [cited 2013 Mar]. Available from: http://journals.lww.com/ optvissci/toc/1995/03000

50. Hazel CA, Elliott DB. The dependency of LogMAR visual acuity measurements on chart design and scoring rule. Optom Vis Sci [serial on the Internet]. 2002;79:788792 [cited 2013 Mar]. Available from: http://journals.lww.com/optvissci/ toc/2002/12000 